\title{
New Inequalities for Gamma and Digamma Functions
}

\author{
M. R. Farhangdoost and M. Kargar Dolatabadi \\ Department of Mathematics, College of Science, Shiraz University, Shiraz 71475-44776, Iran \\ Correspondence should be addressed to M. R. Farhangdoost; farhang@shirazu.ac.ir \\ Received 6 December 2013; Accepted 1 July 2014; Published 12 November 2014 \\ Academic Editor: Vijay Gupta
}

Copyright (C) 2014 M. R. Farhangdoost and M. Kargar Dolatabadi. This is an open access article distributed under the Creative Commons Attribution License, which permits unrestricted use, distribution, and reproduction in any medium, provided the original work is properly cited.

By using the mean value theorem and logarithmic convexity, we obtain some new inequalities for gamma and digamma functions.

\section{Introduction}

Let $\Gamma(x), \psi(x), \psi^{n}(x)$, and $\zeta(x)$ denote the Euler gamma function, digamma function, polygamma functions, and Riemann zeta function, respectively, which are defined by

$$
\begin{gathered}
\Gamma(x)=\int_{0}^{\infty} e^{-t} t^{x-1} d t, \quad \text { for } x>0, \\
\psi(x)=\frac{\Gamma^{\prime}(x)}{\Gamma(x)}, \quad \text { for } x>0,
\end{gathered}
$$

$$
\begin{gathered}
\psi^{(n)}(x) \\
=(-1)^{n+1} \int_{0}^{\infty} \frac{t^{n} e^{-x t}}{1-e^{-t}} d t, \quad \text { for } x>0 ; n=1,2,3, \ldots \\
\zeta(x)=\sum_{n=1}^{\infty} \frac{1}{n^{x}}, \quad \text { for } x>1 .
\end{gathered}
$$

In the past different papers appeared providing inequalities for the gamma, digamma, and polygamma functions (see [118]).

By using the mean value theorem to the function $\log \Gamma(x)$ on $[u, u+1]$, with $x>0$ and $u>0$, Batir [19] presented the following inequalities for the gamma and digamma functions:

$$
\begin{aligned}
& \psi(x) \leqslant \log \left(x-1+e^{-\gamma}\right), \quad \text { for } x>0, \\
& \log (x)-\psi(x)<\frac{1}{2} \psi^{\prime}(x), \text { for } x>1, \\
& \psi^{\prime}(x) \geqslant \frac{\pi^{2}}{6 e^{\gamma}} e^{-\psi(x)}, \quad \text { for } x \geqslant 1 .
\end{aligned}
$$

In Section 2, by applying the mean value theorem on

$$
(\log \Gamma(x))^{\prime}=\psi(x), \quad \text { for } x>0,
$$

we obtain some new inequalities on gamma and digamma functions.

Section 3 is devoted to some new inequalities on digamma function, by using convex properties of logarithm of this function.

Note that in this paper by $\gamma=\lim _{n \rightarrow \infty}\left(\sum_{k=1}^{n}(1 / k)-\right.$ $\log (n))=0.5772156 \cdots$ we mean Euler's constant [5].

\section{Inequalities for Gamma and Digamma Functions by the Mean Value Theorem}

Lemma 1. For $t>0$, one has

$$
\frac{-\psi^{\prime \prime}(t)}{\psi^{\prime}(t)^{2}}<1
$$

Proof. By [6, Proposition 1], we have

$$
\psi^{\prime}(t) \psi^{\prime \prime \prime}(t)-2\left[\psi^{\prime \prime}(t)\right]^{2}<0, \text { for } t>0 \text {. }
$$

Thus the function $\psi^{\prime \prime}(t) / \psi^{\prime}(t)^{2}$ is strictly decreasing on $(0, \infty)$. 
By using asymptotic expansions [20, pages 253-256 and 364],

$$
\begin{aligned}
& \psi^{\prime}(t)=\frac{1}{t}+\frac{1}{2 t^{2}}+\frac{1}{6 t^{3}}+\frac{\theta^{\prime}}{30 t^{5}}, \quad\left(0 \leqslant \theta^{\prime} \leqslant 1\right), \\
& \psi^{\prime \prime}(t)=-\frac{1}{t^{2}}-\frac{1}{t^{3}}-\frac{1}{2 t^{4}}+\frac{1}{6 t^{6}}-\frac{\theta^{\prime \prime}}{6 t^{8}}, \quad\left(0 \leqslant \theta^{\prime \prime} \leqslant 1\right) .
\end{aligned}
$$

For $t>0$, we get

$$
\lim _{t \rightarrow \infty} \frac{\psi^{\prime \prime}(t)}{\psi^{\prime}(t)^{2}}=-1
$$

Now, the proof follows from the monotonicity of $\psi^{\prime \prime}(t) / \psi^{\prime}(t)^{2}$ on $(0, \infty)$ and

$$
\lim _{t \rightarrow \infty} \frac{\psi^{\prime \prime}(t)}{\psi^{\prime}(t)^{2}}=-1
$$

\section{Theorem 2. One has the following:}

(a) $x-(1 / 2)<1 / \psi^{\prime}(x) \leqslant x+\left(6 / \pi^{2}\right)-1$ for $x \geqslant 1$;

(b) $1 / x^{2}<\psi^{\prime}(x) \psi^{\prime}(x+1)<2 / x^{2}$ for $x>0$;

(c) $\left[\psi^{\prime}(x)\right]^{2} / \psi^{\prime \prime}(x) \geqslant-\pi^{4} / 72 \zeta(3)$ for $x \geqslant 1$ and $x^{2} \psi^{\prime}(x+$ 1) $\psi^{\prime}(x)<\pi^{4} / 72 \zeta(3)$ for $x>2$;

(d) $\left(\left[\psi^{\prime}(x+h)\right]^{2}-\psi^{\prime}(x) \psi^{\prime}(x+h)\right) / h \psi^{\prime}(x)>\psi^{\prime \prime}(x+h)$ for $x>0$ and $h>0$;

(e) $\left(\psi^{\prime}(x+h) \psi^{\prime}(x)-\left[\psi^{\prime}(x)\right]^{2}\right) / h \psi^{\prime}(x+h)<\psi^{\prime \prime}(x)$ for $x>0$ and $h>0$;

(f) $-x^{2} \psi^{\prime \prime}(x)<\psi^{\prime}(x) / \psi^{\prime}(x+1)$ and $\psi^{\prime}(x+1) / \psi^{\prime}(x)<$ $-x^{2} \psi^{\prime \prime}(x+1)$ for $x>0$;

(g) $\left(\left(\pi^{2} x / 6\right)+1\right)^{\left(x+\left(6 / \pi^{2}\right)\right)} e^{-x(\gamma+1)} \leqslant \Gamma(x+1)<(2 x+$ $1)^{(x+(1 / 2))} e^{-x(1+\gamma)}$ for $x \geqslant 1$;

(h) $(1 / x)-\psi^{\prime}(x)<(1 / 2) \psi^{\prime \prime}(x+(1 / 2))$ for $x>0$ and $(1 / x)-\psi^{\prime}(x)>\left(\left(\psi^{\prime}\right)^{-1}(1)-1\right) \psi^{\prime \prime}(x)$ for $x>1$;

(i) $\psi(x+1)>\log (x+(1 / 2))+\psi\left(\left(\psi^{\prime}\right)^{-1}(1)\right)$ for $x \geqslant 1 / 2$;

(j) $\left(\pi^{4} / 72 \zeta(3)\right) \log \left(x-\left(\psi^{\prime}\right)^{-1}(1)+2\right)+\psi\left(\left(\psi^{\prime}\right)^{-1}(1)\right) \geqslant$ $\psi(x+1)$ for $x>\left(\psi^{\prime}\right)^{-1}(1)-1$.

Proof. Let $u$ be a positive real number and $\psi(x)$ defined on the closed interval $[u, u+1]$. By using the mean value theorem for the function $\psi(x)$ on $[u, u+1]$ with $u>0$ and since $\psi^{\prime}$ is a decreasing function, there is a unique $\theta$ depending on $u$ such that $0 \leqslant \theta=\theta(u)<1$, for all $u \geqslant 0$; then

$$
\psi(u+1)-\psi(u)=\psi^{\prime}(u+\theta(u))
$$

Since $\psi(x+1)-\psi(x)=1 / x$ and $\psi^{\prime}(x+1)-\psi^{\prime}(x)=-1 / x^{2}$, we have

$$
\psi^{\prime}(u+\theta(u))=\frac{1}{u}, \quad \text { for } u>0
$$

We show that the function $\theta(u)$ has the following properties:

(1) $\theta(u)$ is strictly increasing on $(0, \infty)$;

(2) $\lim _{u \rightarrow \infty} \theta(u)=1 / 2$;

(3) $\theta^{\prime}(u)$ is strictly decreasing on $(0, \infty)$;

(4) $\lim _{u \rightarrow \infty} \theta^{\prime}(u)=0$.

To prove these four properties, since $\psi^{\prime}$ is a decreasing function on $(0, \infty)$, we put $u=1 / \psi^{\prime}(t)$, where $t>0$; by formula (13) we have

$$
\psi^{\prime}\left(\frac{1}{\psi^{\prime}(t)}+\theta\left(\frac{1}{\psi^{\prime}(t)}\right)\right)=\psi^{\prime}(t) .
$$

Since by formula (8) we have $\psi^{\prime \prime}(t)<0$ and $\psi^{\prime}(t)>0$, for all $t>0$, then the mapping $t \rightarrow \psi^{\prime}(t)$ from $(0, \infty)$ into $(0, \infty)$ is injective since also $\psi^{\prime}(t) \rightarrow 0$ and $\psi^{\prime}(t) \rightarrow \infty$ when $t \rightarrow \infty$ and $t \rightarrow 0^{+}$, respectively, then the mapping $t \rightarrow \psi^{\prime}(t)$ from $(0, \infty)$ into $(0, \infty)$ is a bijective map. Clearly, by injectivity of $\psi^{\prime}$, we find that

$$
\theta\left(\frac{1}{\psi^{\prime}(t)}\right)=t-\frac{1}{\psi^{\prime}(t)}, \quad \text { for } t>0 .
$$

Differentiating between both sides of this equation, we get

$$
\theta^{\prime}\left(\frac{1}{\psi^{\prime}(t)}\right)=\frac{-\left[\left(\psi^{\prime}(t)\right)^{2}+\psi^{\prime \prime}(t)\right]}{\psi^{\prime \prime}(t)} .
$$

Since by formula $(8), \psi^{\prime \prime}(t)<0$, where $t>0$, hence formula (15) gives $\theta^{\prime}\left(1 / \psi^{\prime}(t)\right)>0$, for all $t>0$. Since the mapping $t \rightarrow$ $1 / \psi^{\prime}(t)$ from $(0, \infty)$ to $(0, \infty)$ is also bijective, then $\theta^{\prime}(t)>0$ for all $t>0$, and the proof of (1) is completed.

From (8) we have

$$
\begin{aligned}
& \lim _{u \rightarrow \infty} \theta(u) \\
& =\lim _{t \rightarrow \infty} \theta\left(\frac{1}{\psi^{\prime}(t)}\right)=\lim _{t \rightarrow \infty}\left(t-\frac{1}{\psi^{\prime}(t)}\right) \\
& =\lim _{t \rightarrow \infty}\left(t-\frac{1}{(1 / t)+\left(1 / 2 t^{2}\right)+\left(1 / 6 t^{3}\right)+\left(1 / 3 t^{5}\right)}\right) \\
& =\frac{1}{2} .
\end{aligned}
$$

Differentiating between both sides of (15), we obtain

$$
\begin{aligned}
& \theta^{\prime \prime}\left(\frac{1}{\psi^{\prime}(t)}\right) \\
& \quad=\frac{\left[\psi^{\prime}(t)\right]^{3}}{\psi^{\prime \prime}(t)}\left[2\left(\psi^{\prime \prime}(t)\right)^{2}-\psi^{\prime}(t) \psi^{\prime \prime}(t)\right] .
\end{aligned}
$$

Since $\psi^{\prime}(t)>0$ and $\psi^{\prime \prime}(t)<0$, where $t>0$, then $\theta^{\prime \prime}\left(1 / \psi^{\prime}(t)\right)<0$ for all $t>0$. Proceeding as above we conclude that $\theta^{\prime \prime}(t)<0$, for $t>0$. This proves (3). 
For (4), from (8), (9), we conclude that

$$
\begin{aligned}
\lim _{u \rightarrow \infty} \theta^{\prime}(u) & =\lim _{t \rightarrow \infty} \theta^{\prime}\left(\frac{1}{\psi^{\prime}(t)}\right)=\lim _{t \rightarrow \infty}-\frac{\left[\left(\psi^{\prime}(t)\right)^{2}+\psi^{\prime \prime}(t)\right]}{\psi^{\prime \prime}(t)} \\
& =-1-\lim _{t \rightarrow \infty} \frac{\left[\psi^{\prime}(t)\right]^{2}}{\psi^{\prime \prime}(t)}=0 .
\end{aligned}
$$

Now, we prove the theorem. To prove (a), let $1 / \psi^{\prime}(1)=$ $6 / \pi^{2} \leqslant t<\infty$; then by (1) and (2) we have

$$
\theta\left(\frac{1}{\psi^{\prime}(1)}\right) \leqslant \theta(t)<\lim _{t \rightarrow \infty} \theta(t) .
$$

Equation (13) and $\psi^{\prime \prime}(t)<0$ for all $t>0$ give

$$
\theta(t)=\left(\psi^{\prime}\right)^{-1}\left(\frac{1}{t}\right)-t
$$

By substituting the value of $\theta(t)$ into (18), we get

$$
1-\frac{1}{\psi^{\prime}(1)} \leqslant\left(\psi^{\prime}\right)^{-1}\left(\frac{1}{t}\right)-t<\lim _{t \rightarrow \infty} \theta(t)=\frac{1}{2} .
$$

By substituting the value $t=1 / \psi^{\prime}(u)$ into this inequality, we get

$$
u-\frac{1}{2}<\frac{1}{\psi^{\prime}(u)} \leqslant u+\frac{6}{\pi^{2}}-1
$$

where $u \geqslant 1$.

In order to prove (b), by using the mean value theorem on the interval $\left[1 / \psi^{\prime}(t), 1 / \psi^{\prime}(t+1)\right]$, and since $\theta$ is a decreasing function, there exists a unique $\delta$ such that

$$
0<\delta(t)<1
$$

for $t>0$ and

$$
\begin{aligned}
& \theta\left(\frac{1}{\psi^{\prime}(t+1)}\right)-\theta\left(\frac{1}{\psi^{\prime}(t)}\right) \\
& \quad=\left(\frac{1}{\psi^{\prime}(t+1)}-\frac{1}{\psi^{\prime}(t)}\right) \theta^{\prime}\left(\frac{1}{\psi^{\prime}(t+\delta(t))}\right) .
\end{aligned}
$$

Now, by (14), we have

$$
\begin{aligned}
1 & -\frac{1}{\psi^{\prime}(t+1)}+\frac{1}{\psi^{\prime}(t)} \\
& =\left(\frac{1}{\psi^{\prime}(t+1)}-\frac{1}{\psi^{\prime}(t)}\right) \theta^{\prime}\left(\frac{1}{\psi^{\prime}(t+\delta(t))}\right) .
\end{aligned}
$$

Since $\theta$ is strictly increasing on $(0, \infty)$, by $(1)$, we have

$$
\begin{aligned}
1 & +\frac{\psi^{\prime}(t+1)-\psi^{\prime}(t)}{\psi^{\prime}(t+1) \psi^{\prime}(t)} \\
& =\theta\left(\frac{1}{\psi^{\prime}(t+1)}\right)-\theta\left(\frac{1}{\psi^{\prime}(t)}\right)>0 .
\end{aligned}
$$

By using this inequality and the fact that $\psi(x+1)-\psi(x)=1 / x$ and

$$
\psi^{\prime}(x+1)-\psi^{\prime}(x)=-\frac{1}{x^{2}},
$$

we obtain

$$
\psi^{\prime}(t+1) \psi^{\prime}(t)>\frac{1}{t^{2}}, \quad t>0 .
$$

Since $\theta$ is strictly increasing on $(0, \infty)$, by $(1)$, it is clear that

$$
\begin{aligned}
& \theta\left(\frac{1}{\psi^{\prime}(t+1)}\right)-\theta\left(\frac{1}{\psi^{\prime}(t)}\right) \\
& \quad<\lim _{t \rightarrow \infty} \theta(t)-\theta\left(0^{+}\right)=\frac{1}{2}, \quad t>0 .
\end{aligned}
$$

and then it is clear that (b) holds.

For (c), since $t>2, t+\delta(t)>1+\delta(1)$, and $\theta^{\prime}$ is strictly decreasing on $(0, \infty)$ by $(3)$, then

$$
\begin{array}{r}
\theta^{\prime}\left(\frac{1}{\psi^{\prime}(t+\delta(t))}\right)<\theta^{\prime}\left(\frac{1}{\psi^{\prime}(1)}\right)=-1-\frac{\left[\psi^{\prime}(1)\right]^{2}}{\psi^{\prime \prime}(1)}, \\
\forall t>2 .
\end{array}
$$

Since $\psi(x+1)-\psi(x)=1 / x$ and $\psi^{\prime}(x+1)-\psi^{\prime}(x)=-1 / x^{2}$, by using (24), we obtain

$$
t^{2} \psi^{\prime}(t+1) \psi^{\prime}(t)<\frac{\pi^{4}}{72 \zeta(3)},
$$

where $t>2$.

Since $\theta^{\prime}$ is strictly decreasing on $(0, \infty)$ by $(3)$ and $\psi^{\prime \prime}(t)<$ 0 , for all $t>0$, we have

$$
\theta^{\prime}\left(\frac{1}{\psi^{\prime}(t)}\right) \leqslant \theta^{\prime}\left(\frac{1}{\psi^{\prime}(1)}\right)
$$

where $t \geqslant 1$.

Then it is clear that (c) is true.

Now we prove (d) and (e) by using the mean value theorem on $\left[1 / \psi^{\prime}(t), 1 / \psi^{\prime}(t+h)\right](t>0, h>0)$, for $\theta$, we conclude

$$
\begin{aligned}
& \theta\left(\frac{1}{\psi^{\prime}(t+h)}\right)-\theta\left(\frac{1}{\psi^{\prime}(t)}\right) \\
& \quad=\left(\frac{1}{\psi^{\prime}(t+h)}-\frac{1}{\psi^{\prime}(t)}\right) \theta^{\prime}\left(\frac{1}{\psi^{\prime}(t+a)}\right),
\end{aligned}
$$

where $0<a<h$.

After brief computation we have

$$
\theta^{\prime}\left(\frac{1}{\psi^{\prime}(t+a)}\right)=\frac{h \psi^{\prime}(t+h) \psi^{\prime}(t)}{\psi^{\prime}(t)-\psi^{\prime}(t+h)}-1, \quad t>0 .
$$

Since $t+a>t$ for all $a>0, t>0$, and by the monotonicity of $\theta^{\prime}$ and $\psi^{\prime}$ we have $\theta^{\prime}\left(1 / \psi^{\prime}(t+a)\right)<\theta^{\prime}\left(1 / \psi^{\prime}(t)\right)$; then

$$
\frac{\psi^{\prime}(t+h) \psi^{\prime}(t)-\left[\psi^{\prime}(t)\right]^{2}}{h \psi^{\prime}(t+h)}<\psi^{\prime \prime}(t), \quad t>0, h>0 .
$$


By monotonicity of $\theta^{\prime}$ and $\psi^{\prime}$, we have

$$
\theta^{\prime}\left(\frac{1}{\psi^{\prime}(t+a)}\right)>\theta^{\prime}\left(\frac{1}{\psi^{\prime}(t+h)}\right)
$$

After some simplification of this inequality $(\mathrm{d})$ is proved.

For (f), we put $h=1$ in (e) and (d).

For $(\mathrm{g})$, we integrate (a) on $[1, t]$ for $t>0$; then we have

$$
\begin{aligned}
& \log \left(\frac{(t-1) \pi^{2}}{6}+1\right)-\gamma \\
& \quad \leqslant \psi(t)<\log (2 t-1)-\gamma, \text { for } t \geqslant 1 ;
\end{aligned}
$$

the proof is completed when we integrate these inequalities on $[1, s]$, for $s>0$.

By using the mean value theorem for the $\psi^{\prime}(t)$ on $[t, t+$ $\theta(t)]$, there is a $\alpha(t)$ depending on $t$ such that $0<\alpha(t)<\theta(t)$ for all $t>0$, and so

$$
\psi^{\prime}(t+\theta(t))=\theta(t) \psi^{\prime \prime}(t+\alpha(t))+\psi^{\prime}(t) .
$$

By formula (13) and (2), since $\psi^{\prime \prime}$ is strictly increasing on $(0, \infty)$, we have

$$
\begin{aligned}
& \psi^{\prime \prime}(t+\alpha(t)) \theta(t) \\
& =\frac{1}{t}-\psi^{\prime}(t)<\lim _{t \rightarrow \infty} \theta(t) \psi^{\prime \prime}\left(t+\lim _{t \rightarrow \infty} \theta(t)\right), \quad \text { for } t>0
\end{aligned}
$$

or

$$
\frac{1}{t}-\psi^{\prime}(t)<\frac{1}{2} \psi^{\prime \prime}\left(t+\frac{1}{2}\right), \quad \text { for } t>0 ;
$$

since $\psi^{\prime \prime}$ is strictly increasing on $(0, \infty)$, by $(1)$, we have

$$
\theta(t) \psi^{\prime \prime}(t+\alpha(t))=\frac{1}{t}-\psi^{\prime}(t)>\theta(1) \psi^{\prime \prime}(t)
$$

or

$$
\frac{1}{t}-\psi^{\prime}(t)>\left(\left(\psi^{\prime}\right)^{-1}(1)-1\right) \psi^{\prime \prime}(t), \quad \text { for } t>1 .
$$

In order to prove (i) and (j), we integrate both sides of (13) over $1 \leqslant u \leqslant x$ to obtain

$$
\int_{1}^{x} \psi^{\prime}(u+\theta(u)) d u=\int_{1}^{x} \frac{1}{u} d u
$$

Making the change of variable $u=1 / \psi^{\prime}(t)$ on the left-hand side, by (14), we have

$$
\int_{\left(\psi^{\prime}\right)^{-1}(1)}^{x+\theta(x)} \psi^{\prime}(t) \frac{-\psi^{\prime \prime}(t)}{\psi^{\prime}(t)^{2}} d t=\log (x) ;
$$

since $\psi^{\prime}(t)>0$ for all $t>0$ and $\psi^{\prime}(x) \psi^{\prime \prime}(x)-2\left[\psi^{\prime \prime}(x)\right]^{2}<0$, we find that, for $x>1$,

$$
\begin{aligned}
\log (x) & <\int_{\left(\psi^{\prime}\right)^{-1}(1)}^{x+\theta(x)} \psi^{\prime}(t) d t \\
& =\psi(x+\theta(x))-\psi\left(\left(\psi^{\prime}\right)^{-1}\right.
\end{aligned}
$$

or

$$
\log (x)+\psi\left(\left(\psi^{\prime}\right)^{-1}(1)\right)<\psi(x+\theta(x))
$$

Again using the monotonicity of $\theta$ and $\psi$, after some simplifications as for $x \geqslant 1 / 2$, we can rewrite

$$
\log \left(x+\frac{1}{2}\right)+\psi\left(\left(\psi^{\prime}\right)^{-1}(1)\right)<\psi(x+1) .
$$

This proves (i). By inequality (c) for $x \geqslant 1$, we have

$$
\begin{aligned}
& \log (x) \geqslant \frac{72 \zeta(3)}{\pi^{4}} \int_{\left(\psi^{\prime}\right)^{-1}(1)}^{x+\theta(x)} \psi^{\prime}(t) d t \\
& =\frac{72 \zeta(3)}{\pi^{4}}\left(\psi(x+\theta(x))-\psi\left(\left(\psi^{\prime}\right)^{-1}(1)\right)\right) ;
\end{aligned}
$$

since for $\left.x \geqslant 1, \theta(x) \geqslant \theta(1)=\left(\left(\psi^{\prime}\right)^{-1}(1)-1\right)\right)=\left(\psi^{\prime}\right)^{-1}(1)-1$, from this inequality we find that

$$
\begin{gathered}
\frac{\pi^{4}}{72 \zeta(3)} \log (x)+\psi\left(\left(\psi^{\prime}\right)^{-1}(1)\right) \\
\geqslant \psi\left(x+\left(\psi^{\prime}\right)^{-1}(1)-1\right)
\end{gathered}
$$

replacing $x$ by $x-\left(\psi^{\prime}\right)^{-1}(1)+2$, we get for $x \geqslant\left(\psi^{\prime}\right)^{-1}(1)-1$

$$
\begin{aligned}
& \frac{\pi^{4}}{72 \zeta(3)} \log \left(x-\left(\psi^{\prime}\right)^{-1}(1)+2\right)+\psi\left(\left(\psi^{\prime}\right)^{-1}(1)\right) \\
& \geqslant \psi(x+1)
\end{aligned}
$$

which proves $(\mathrm{j})$. Then the proof is completed.

Example 3. Consider the matrix

$$
A_{n}=\left[\begin{array}{ccccc}
3 & 1 & 1 & \cdots & 1 \\
1 & 4 & 1 & \cdots & 1 \\
& & \vdots & & \\
1 & 1 & \cdots & 1 & n+1
\end{array}\right] .
$$

By using inequalities (a), we obtain

$$
\frac{\pi^{2}}{\pi^{2} x+6-\pi^{2}} \leqslant \psi^{\prime}(x)<\frac{2}{2 x-1}, \quad x \geqslant 1 .
$$

Now, we integrate on $[1, t]$ (for $t>0$ ) from both sides of $(51)$ to obtain

$$
\log \left(\frac{(t-1) \pi^{2}}{6}+1\right)-\gamma \leqslant \psi(t)<\log (2 t-1)-\gamma
$$

replacing $t$ by $n+1$ ( $n$ is an integer number) and using the identity $\psi(n+1)=H_{n}-\gamma[6]$ and $\operatorname{det} A_{n}=n ! H_{n}$ [21], where $H_{n}=\sum_{k=1}^{n}(1 / k)$ is the $n$th harmonic number, then we have

$$
\log \left(\frac{n \pi^{2}}{6}+1\right)^{n !} \leqslant n ! H_{n}<\log (2 n+1)^{n !} .
$$




\section{New Inequalities for Digamma Function by Properties of Strictly Logarithmically Convex Functions}

Definition 4. A positive function $f$ is said to be logarithmically convex on an interval $I$ if $f$ has derivative of order two on $I$ and

$$
(\log f(x))^{\prime \prime} \geqslant 0
$$

for all $x \in I$.

If inequality (54) is strict, for all $x \in I$, then $f$ is said to be strictly logarithmically convex [22].

Lemma 5. The function $\Gamma$ is increasing on $[c, \infty)$, where $c=$ $1 / 46163 \cdots$ is the only positive zero of $\psi[1,19]$.

Lemma 6. If $x \geqslant c$ and $k(x)=1 / \psi(x)$, then $k$ is strictly logarithmically convex on $[c, \infty)$.

Proof. By differentiation we have

$$
[\log k(x)]^{\prime \prime}=\left[\frac{-\psi^{\prime}(x)}{\psi(x)}\right]^{\prime}=\frac{-\psi^{\prime \prime}(x) \psi(x)+\left[\psi^{\prime}(x)\right]^{2}}{[\psi(x)]^{2}}
$$

by Lemma 5 , we obtain $\psi(x)=\Gamma^{\prime}(x) / \Gamma(x)>0$, for every $x \in[c, \infty)$ and since $\psi^{\prime \prime}(x)<0$ on $(0, \infty)$, then we have $(\log k(x))^{\prime \prime}>0$, for $x \geqslant c$.

This implies that $1 / \psi(x)$ is strictly logarithmically convex on $[c, \infty)$.

Theorem 7. One has the following:

(a) $[\psi(x+3)]^{a} / \psi(a x+3)>((3 / 2)-\gamma)^{a-1}$, for $a>1$ and $x>-3 / a$

(b) $[\psi(x+3)]^{a} / \psi(a x+3)<((11 / 6)-\gamma)^{a} / \psi(3+a)$, for $a>1$ and $x \in(0,1)$;

(c) $[\psi(x+3)]^{a} / \psi(a x+3)>((11 / 6)-\gamma)^{a} / \psi(3+a)$, for $a>1$ and $x>1$;

(d) $[\psi(x+3)]^{a} / \psi(a x+3)>((11 / 6)-\gamma)^{a} / \psi(3+a)$, for $a \in(0,1)$ and $x \in(0,1)$;

(e) $[\psi(x+3)]^{a} / \psi(a x+3)<((11 / 6)-\gamma)^{a} / \psi(3+a)$, for $a \in(0,1)$ and $x>1$.

Proof. By Lemma 6 we have, for $a>1$,

$$
\psi\left[\frac{u}{p}+\frac{v}{q}\right]>[\psi(u)]^{1 / p}[\psi(v)]^{1 / q}
$$

where $p>1, q>1,(1 / p)+(1 / q)=1, u \geqslant c$, and $v \geqslant c$.

If $p=a$ and $q=a /(a-1)$, then

$$
\psi\left[\frac{1}{a} u+\left(1-\frac{1}{a}\right) v\right]>[\psi(u)]^{1 / a}[\psi(v)]^{1-(1 / a)}
$$

for $u \geqslant c$ and $v \geqslant c$.
Let $v=3$ and $u=a x+3$. Note that $\psi(3)=(3 / 2)-\gamma$ and $(1 / a) u+(1-(1 / a)) v=x+3$; also we obtain

$$
\frac{[\psi(x+3)]^{a}}{\psi(a x+3)}>\left(\frac{3}{2}-\gamma\right)^{a-1} \text { for } x=\frac{u-3}{a}>-\frac{3}{a} .
$$

In order to prove (b), let

$$
\begin{aligned}
f(x)= & \log \psi(a x+3)-\log \psi(3+a) \\
& -a \log \psi(x+3) ;
\end{aligned}
$$

since $\psi(4)=(11 / 6)-\gamma$, we have $f(1)=\log ((11 / 6)-\gamma)^{-a}$. Also

$$
f^{\prime}(x)=a\left[\frac{\psi^{\prime}(a x+3)}{\psi(a x+3)}-\frac{\psi^{\prime}(x+3)}{\psi(x+3)}\right] .
$$

By Lemma $6, \log (1 / \psi(t))$ is strictly convex on $[c, \infty)$; then $(\log \psi(t))^{\prime \prime}<0$ and so $\left(\psi^{\prime}(t) / \psi(t)\right)^{\prime}<0$; this implies that $\left(\psi^{\prime}(t) / \psi(t)\right)$ is strictly decreasing on $[c, \infty)$. Since $a>1$ and $x \in(0,1)$, we have $a x+3>x+3$. Then

$$
\frac{\psi^{\prime}(a x+3)}{\psi(a x+3)}<\frac{\psi^{\prime}(x+3)}{\psi(x+3)} \text {. }
$$

And then $f^{\prime}(x)<0$; also $f(1)=\log ((11 / 6)-\gamma)^{-a}$. Then

$$
f(x)>f(1)=\log \left(\frac{11}{6}-\gamma\right)^{-a}
$$

for $a>1$ and $x \in(0,1)$ or

$$
\frac{[\psi(x+3)]^{a}}{\psi(a x+3)}<\frac{((11 / 6)-\gamma)^{a}}{\psi(3+a)} .
$$

So (b) is proved.

By

$$
\begin{aligned}
& a x+3>x+3, \quad \text { for } a>1, x>1, \\
& a x+3<x+3, \quad \text { for } a \in(0,1), x \in(0,1), \\
& a x+3<x+3, \quad \text { for } a \in(0,1), x>1,
\end{aligned}
$$

(c), (d), and (e) are clear.

Corollary 8. For all $x \in(0,1)$ and all integers $n>1$, one has

$$
\left(\frac{3}{2}-\gamma\right)^{n-1}<\frac{[\psi(x+3)]^{n}}{\psi(n x+3)}<\frac{((11 / 6)-\gamma)^{n}}{H_{n+2}-\gamma}
$$

where $H_{n}=\sum_{k=1}^{n}(1 / k)$ is the nth harmonic number.

Proof. By [6], for all integers $n \geqslant 1$, we have

$$
\psi(n+1)=H_{n}-\gamma
$$

and replacing $a$ by $n$ in Theorem 7, the proof is completed. 
Theorem 9. Let $f$ be a function defined by

$$
f(x)=\frac{[\psi(3+b x)]^{a}}{[\psi(3+a x)]^{b}} ; \quad \forall x>0,
$$

where $3+a x \geqslant c$ and $3+b x \geqslant c$; then for all $a>b>0$ or $0>a>b(a>0$ and $b<0), f$ is strictly increasing (strictly decreasing $)$ on $(0, \infty)$.

Proof. Let $g$ be a function defined by

$$
g(x)=\log f(x)=a \log \psi(3+b x)-b \log \psi(3+a x) ;
$$

then

$$
g^{\prime}(x)=a b\left[\frac{\psi^{\prime}(3+b x)}{\psi(3+b x)}-\frac{\psi^{\prime}(3+a x)}{\psi(3+a x)}\right] .
$$

By proof of Theorem 7, we have

$$
(\log \psi(x))^{\prime \prime}<0, \quad \text { for } x \in[c, \infty)
$$

this implies that $g^{\prime}(x)>0$ if $a>b>0$ or $0>a>b\left(g^{\prime}(x)<0\right.$ if $a>0$ and $b<0)$; that is, $g$ is strictly increasing on $(0, \infty)$ (strictly decreasing on $(0, \infty)$ ). Hence $f$ is strictly increasing on $(0, \infty)$, if $a>b>0$ or $0>a>b$ (strictly decreasing if $a>0$ and $b<0)$.

Corollary 10. For all $x \in(0,1)$ and all $a>b>0$ or $0>a>b$, one has

$$
\left(\frac{3}{2}-\gamma\right)^{a-b}<\frac{[\psi(3+b x)]^{a}}{[\psi(3+a x)]^{b}}<\frac{[\psi(3+b)]^{a}}{[\psi(3+a)]^{b}}
$$

where $3+b x \geqslant c, 3+a x \geqslant c, 3+b \geqslant c$, and $3+a \geqslant c$.

Proof. To prove (71), applying Theorem 9 and taking account of $\psi(3)=(3 / 2)-\gamma$, we get $f(0)<f(x)<f(1)$ for all $x \in$ $(0,1)$, and we obtain $(71)$.

Corollary 11. For all $x \in(0,1)$ and all $a>0$ and $b<0$, one has

$$
\frac{[\psi(3+b)]^{a}}{[\psi(3+a)]^{b}}<\frac{[\psi(3+b x)]^{a}}{[\psi(3+a x)]^{b}}<\left(\frac{3}{2}-\gamma\right)^{a-b}
$$

where $3+a x \geqslant c, 3+b x \geqslant c, 3+b \geqslant c$, and $3+a \geqslant c$.

Proof. Applying Theorem 9, we get $f(1)<f(x)<f(0)$ for all $x \in(0,1)$, and we obtain $(72)$.

Corollary 12. For all $x \in(0,1)$ and all $a>b>0$ or $0>a>b$, one has

$$
\frac{[\psi(3+b y)]^{a}}{[\psi(3+a y)]^{b}}<\frac{[\psi(3+b x)]^{a}}{[\psi(3+a x)]^{b}}
$$

where $3+a x \geqslant c, 3+b x \geqslant c, 3+a y \geqslant c, 3+b y \geqslant c$, and $0<y<x<1$.
Corollary 13. For all $x \in(0,1)$ and all $a>0$ and $b<0$, one has

$$
\frac{[\psi(3+b x)]^{a}}{[\psi(3+a x)]^{b}}<\frac{[\psi(3+b y)]^{a}}{[\psi(3+a y)]^{b}},
$$

where $3+a x \geqslant c, 3+b x \geqslant c, 3+a y \geqslant c, 3+b y \geqslant c$, and $0<y<x<1$.

Remark 14. Taking $a=n$ and $b=1$ in Corollary 10 , we obtain inequalities of Corollary 8.

\section{Conflict of Interests}

The authors declare that there is no conflict of interests regarding the publication of this paper.

\section{References}

[1] H. Alzer and S. Ruscheweyh, "A subadditive property of the gamma function," Journal of Mathematical Analysis and Applications, vol. 285, no. 2, pp. 564-577, 2003.

[2] H. Alzer, "Some gamma function inequalities," Mathematics of Computation, vol. 60, no. 201, pp. 337-346, 1993.

[3] A. V. Boyd, "Gurland's inequality for the gamma function," Scandinavian Actuarial Journal, vol. 1960, pp. 134-135, 1960.

[4] J. Bustoz and M. E. H. Ismail, "On gamma function inequalities," Mathematics of Computation, vol. 47, no. 176, pp. 659-667, 1986.

[5] C. P. Chen, "Asymptotic expansions of the logarithm of the gamma function in the terms of the polygamma functions," Mathematical Inequalities and Applications, vol. 2, pp. 521-530, 2014.

[6] C.-P. Chen, "Some properties of functions related to the gamma, psi and tetragamma functions," Computers \& Mathematics with Applications, vol. 62, no. 9, pp. 3389-3395, 2011.

[7] C. P. Chen, "Unified treatment of several asymptotic formulas for the gamma function," Numerical Algorithms, vol. 64, no. 2, pp. 311-319, 2013.

[8] T. Erber, "The gamma function inequalities of Gurland and Gautschi," vol. 1960, no. 1-2, pp. 27-28, 1960.

[9] J. D. Keckic and P. M. Vasic, "Some inequalities for the gamma function," Publications de l'Institut Mathématique, vol. 11, pp. 107-114, 1971.

[10] A. Laforgia, "Further inequalities for the gamma function," Mathematics of Computation, vol. 42, no. 166, pp. 597-600, 1984.

[11] D. Lu and X. Wang, "A new asymptotic expansion and some inequalities for the gamma function," Journal of Number Theory, vol. 140, pp. 314-323, 2014.

[12] C. Mortici, "A continued fraction approximation of the gamma function," Journal of Mathematical Analysis and Applications, vol. 402, no. 2, pp. 405-410, 2013.

[13] C. Mortici, "Best estimates of the generalized Stirling formula," Applied Mathematics and Computation, vol. 215, no. 11, pp. 4044-4048, 2010.

[14] C. Mortici, V. G. Cristea, and D. Lu, "Completely monotonic functions and inequalities associated to some ratio of gamma function," Applied Mathematics and Computation, vol. 240, pp. 168-174, 2014.

[15] C. Mortici, "Estimating gamma function by digamma function," Mathematical and Computer Modelling, vol. 52, no. 5-6, pp. 942946, 2010. 
[16] C. Mortici, "New approximation formulas for evaluating the ratio of gamma functions," Mathematical and Computer Modelling, vol. 52, no. 1-2, pp. 425-433, 2010.

[17] C. Mortici, "New improvements of the Stirling formula," Applied Mathematics and Computation, vol. 217, no. 2, pp. 699-704, 2010.

[18] C. Mortici, "Ramanujan formula for the generalized Stirling approximation," Applied Mathematics and Computation, vol. 217, no. 6, pp. 2579-2585, 2010.

[19] N. Batir, "Some new inequalities for gamma and polygamma functions," Journal of Inequalities in Pure and Applied Mathematics, vol. 6, no. 4, article 103, 2005.

[20] B. J. English and G. Rousseau, "Bounds for certain harmonic sums," Journal of Mathematical Analysis and Applications, vol. 206, no. 2, pp. 428-441, 1997.

[21] T. P. Dence and J. B. Dence, "A survey of Euler's constant," American Mathematical Monthly, vol. 82, pp. 225-265, 2009.

[22] E. Artin, The Gamma Function (Trans M. Butter), Holt, Rinehart and Winston, San Francisco, Calif, USA, 1964. 


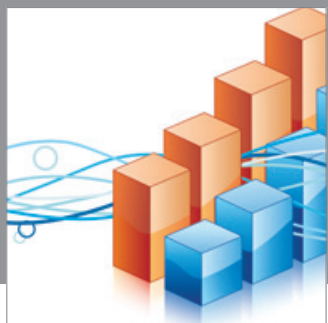

Advances in

Operations Research

mansans

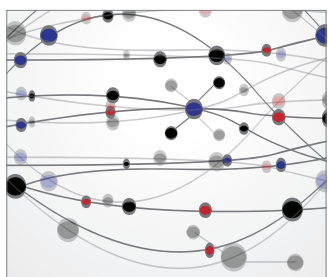

The Scientific World Journal
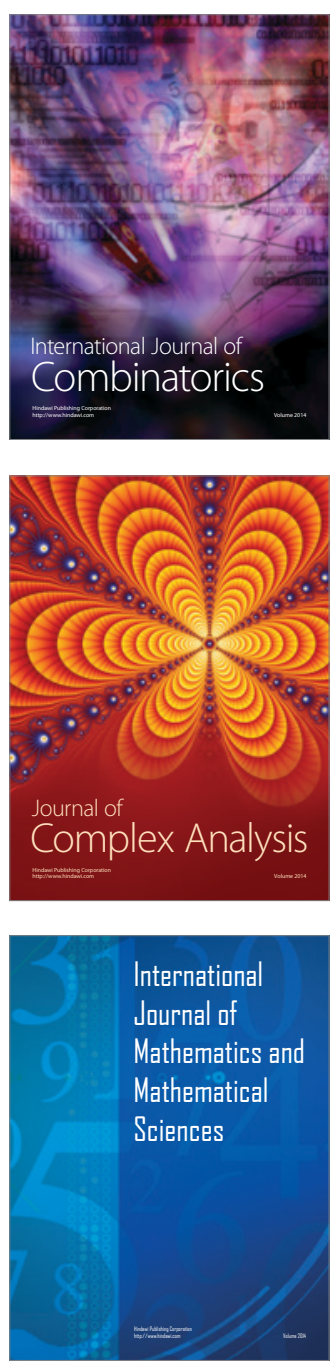
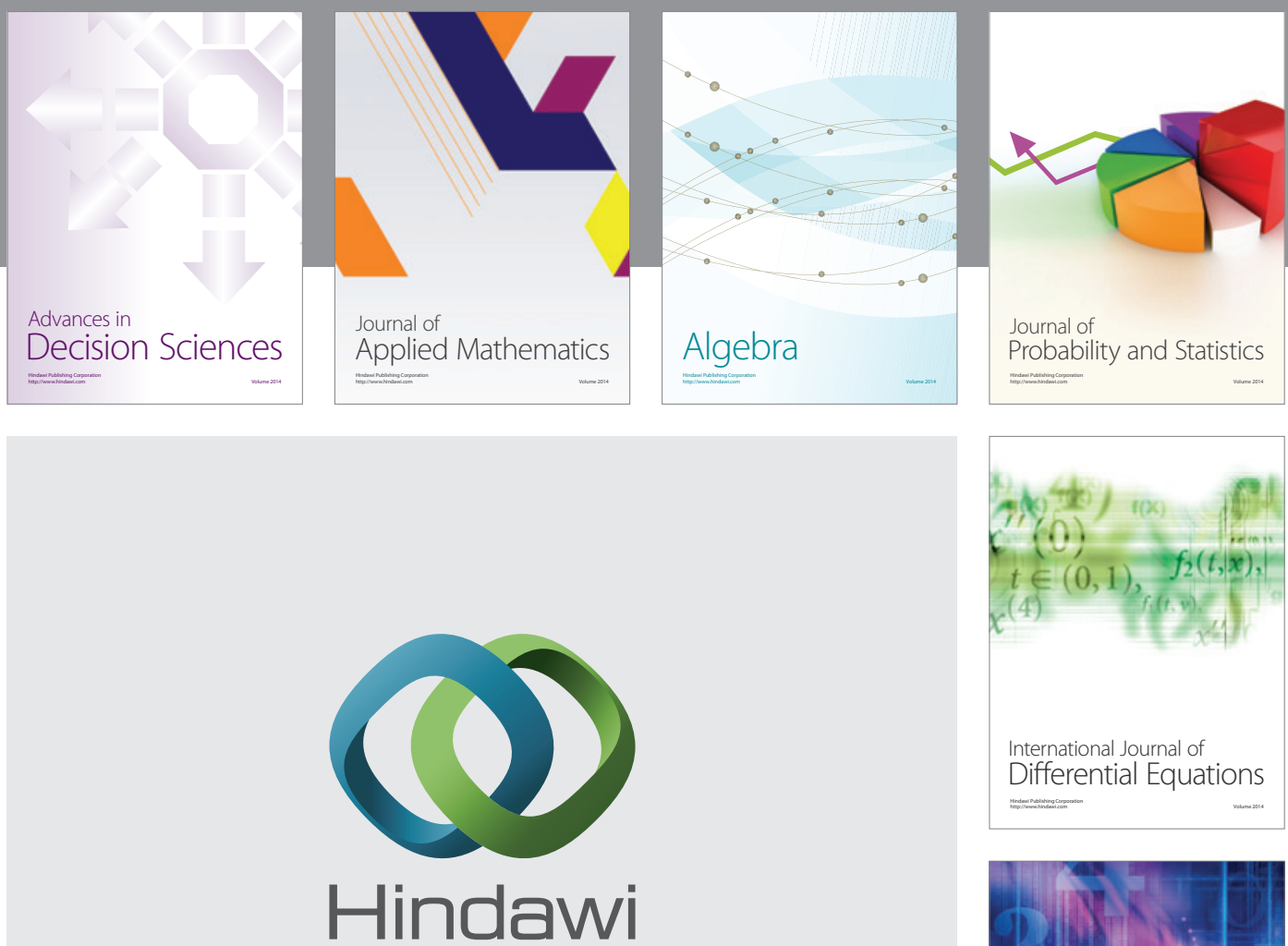

Submit your manuscripts at http://www.hindawi.com
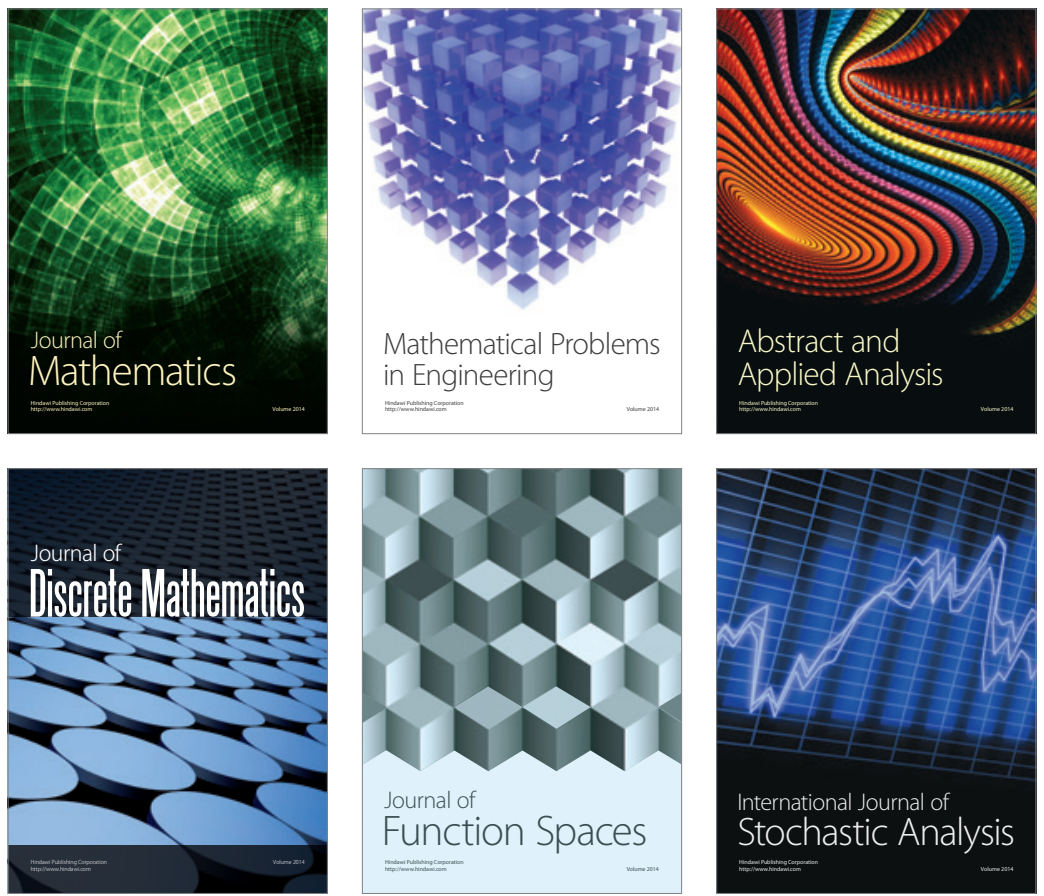

Journal of

Function Spaces

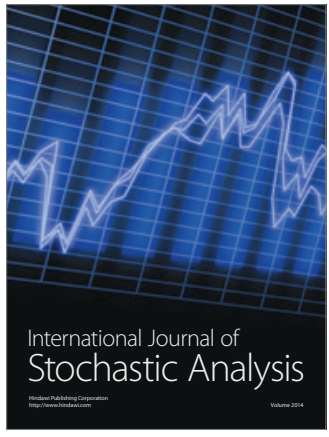

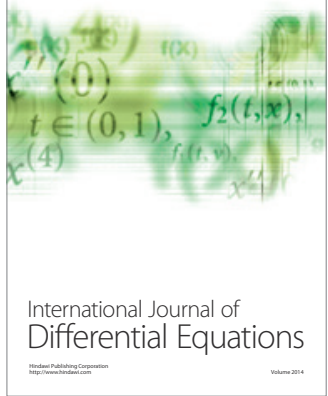
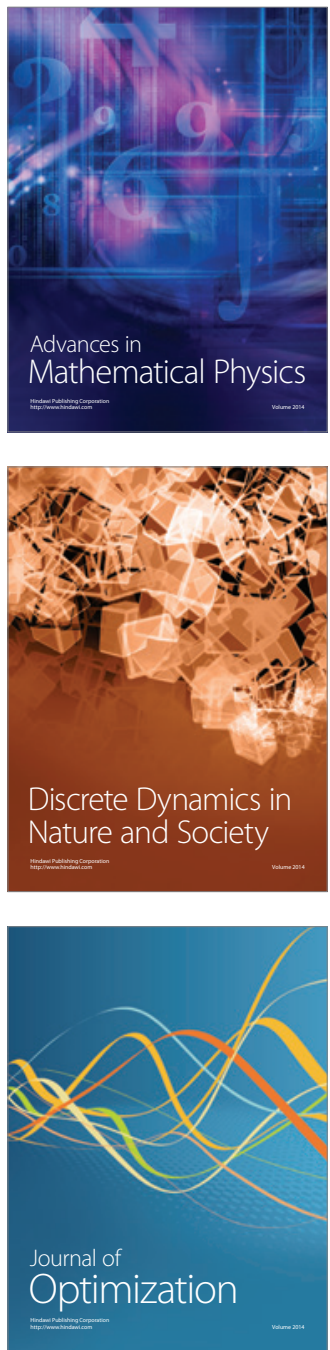